\title{
THE EFFECTS OF NONEQUILIBRIUM HEAT, MASS AND MOMENTUM TRANSFER ON TWO-PHASE SOUND SPEED
}

\author{
R. C. MECREDY and L. J. HAMILTON \\ Nuclcar Engineering Department. University of Michigan, Ann Arbor, Michigan. U.S.A.
}

(Received 31 December 1970 and in revised form 16 April 1971)

\begin{abstract}
A model has been developed which yields the speed and attenuation of acoustic waves as a function of lrequency in two-phase, one-component media. Nonequilibrium interphase heat, mass, and momentum transfer have been included and are found to be important. At low frequencies, the wave speed approaches the equilibrium value calculated by $\sqrt{ }(\partial p / \partial p)_{s}$. The wave speed in the high frequency limit is characterized by no heat, mass. or momentum transfer between the liquid and vapor phases. The attenuation is large at high frequencies. A comparison of the theory with experiment shows that the leading edges of large amplitude pressure waves travel at the high frequency limit of the sound speed.
\end{abstract}

\section{NOMENCLATURE}

$a, \quad$ bubble or droplet radius [ $\mathrm{ft}$ :

c, $\quad$ sound speed $[\mathrm{ft} / \mathrm{s}]$ :

$c_{\mathrm{ad}}, \quad$ adiabatic sound speed [ft/s];

$c_{\text {eq }}, \quad$ equilibrium sound speed [ft/s]:

$\mathcal{c}_{\mathrm{HI}}, \quad$ high frequency limit of the sound speed $[\mathrm{ft} / \mathrm{s}]$ :

$c_{\text {iso }}, \quad$ isothermal sound speed, [ $\left.\mathrm{ft} / \mathrm{s}\right]$ :

$c_{l}$, specific heat-pure liquid phase [Btu/ $\left.\mathrm{lb}^{\circ} \mathrm{F}\right]$;

$c_{p_{g}}$, specific heat, constant pressurepure vapor phase $\left[\mathrm{Btu} / \mathrm{lb}^{\circ} \mathrm{F}\right]$;

$c_{v_{g}}, \quad$ specific heat, constant volume-pure vapor phase $\left[\mathrm{Btu} / \mathrm{bb}^{\circ} \mathrm{F}\right]$ :

$\mathrm{D} / \mathrm{D} t^{3}$, substantial derivative $\left(\mathrm{D} / \mathrm{D} t^{3} \equiv\right.$ $\partial / \partial t+v_{q} \partial / \partial z$ ), equation (3);

$F_{B}, \quad$ force on a bubble due to relative motion of the liquid, equation (14):

$F^{g l}$, inverse time constant for momentum transfer, liquid to vapor $[1 / \mathrm{s}]$ :

$F^{g^{\prime}}, \quad F^{g^{\prime}} \equiv F^{g l}+M / \rho_{g}$, equation (19);

$F^{i g}, \quad$ inverse time constant for momentum transfer, vapor to liquid $[1 / \mathrm{s}]$ :

$G^{g l}$, inverse time constant for heat transfer, liquid to vapor $[1 / \mathrm{s}]$ :
$G^{g l^{\prime}}, \quad G^{g l^{\prime}} \equiv G^{g l}+M\left[\varepsilon_{2} c_{p_{g}}+\varepsilon_{1} c_{l}\right] / \rho_{g} c_{n_{g}}$ equation (19):

$G_{l g}$, inverse time constant for heat transfer, vapor to liquid, [1/s]:

$G_{0}^{g l}, \quad$ low frequency limit of $G^{g l}$;

$h_{f g}(T)$, heat of vaporization evaluated at saturation temperature $T[\mathrm{Btu} / \mathrm{lb}]$ :

$k, \quad$ complex wave number $[k \equiv(\omega / c)+$ in] $[1 / \mathrm{ft}]$ :

$K_{g}$, thermal diffusivity-pure vapor phase, $\left[\mathrm{ft}^{2} / \mathrm{s}\right]$ :

$M, \quad M \equiv 3 \alpha \sigma p / a \sqrt{ } 2 \pi R T$, equation (19);

$N, \quad$ number of bubbles per unit volume of mixture $\left[1 / \mathrm{ft}^{3}\right]$ :

$n, \quad n \equiv\left(\delta p^{*} / p\right)\left(T / \delta T_{l}\right)=h_{f_{g}} / p v_{f g}$, equation (19):

$p, \quad$ pressure [psia] ;

$p^{*}, \quad$ saturation pressure corresponding to temperature $T_{l}$ [psia] :

$R$, gas constant, pure vapor phase $\left[\mathrm{Btu} / \mathrm{h} \mathbf{b}^{\circ} \mathrm{F}\right]$ :

$t, \quad$ time coordinate [s];

$T$, temperature, equilibrium mixture $\left[{ }^{\circ} \mathrm{F}\right]$ :

$T_{g}, \quad$ temperature, vapor phase $\left[{ }^{\circ} \mathrm{F}\right]$ : 
$T_{l}, \quad$ temperature, liquid phase $\left[{ }^{\circ} \mathrm{F}\right]$ :

$U, \quad$ relative velocity of vapor with respect to liquid $[\mathrm{ft} / \mathrm{s}]$ :

$U_{f g}$, change in internal energy upon evaporation $[\mathrm{Btu} / \mathrm{lb}]$ :

$U_{g}(T)$, internal energy of the vapor at saturation temperature $T,[\mathrm{Btu} / \mathrm{lb}]$ :

$U_{l}(T)$, internal energy of the liquid at saturation temperature $T,[\mathrm{Btu} / \mathrm{lb}]$ :

$v_{f y}$, change in specific volume upon evaporation, $\left[\mathrm{ft}^{3} / \mathrm{lb}\right]$ :

$v_{g}, \quad$ velocity, vapor $[\mathrm{ft} / \mathrm{s}]$ :

$v_{l}, \quad$ velocity, liquid $[\mathrm{ft} / \mathrm{s}]$ :

$z$. spatial coordinate $[\mathrm{ft}]$ :

$\alpha, \quad$ void fraction [dimensionless]:

$\beta, \quad$ complex constant, equation (18a):

$\gamma, \quad$ specific heat ratio, pure vapor phase $\left(\gamma=c_{p_{g}} / c_{v_{g}}\right)$;

$\Gamma_{c}$. rate of condensation per unit volume of mixture [ $\left.\mathrm{lb} / \mathrm{ft}^{3} \mathrm{~s}\right]$ :

$\Gamma_{e}, \quad$ rate of evaporation per unit volume of mixture $\left[\mathrm{lb} / \mathrm{ft}^{3} \mathrm{~s}\right]$ :

$\varepsilon_{1}, \quad \varepsilon_{1} \equiv \rho_{g} c_{p_{g}}\left(\rho_{l} \mathcal{c}_{l}+\rho_{g} c_{p_{g}}\right)$, equation (19);

$\varepsilon_{2}, \quad \varepsilon_{2} \equiv \rho_{l} c_{l} /\left(\rho_{l} c_{l}+\rho_{g} c_{p_{g}}\right), \quad$ equation (19):

$\eta$ wave attenuation coefficient $[1 / \mathrm{ft}]$ : $\mu, \quad$ viscosity, pure liquid phase $\left[\mathrm{lb} / \mathrm{s} / \mathrm{ft}^{2}\right]$ :

$\rho_{\mathrm{AP}}, \quad$ apparent mass contribution to vapor mass in bubbly mixtures $\left(\rho_{\mathrm{AP}}=\right.$ $\left.\frac{1}{2} \alpha \bar{\rho}_{l}(1+2 \alpha / 1-\alpha)\right)\left[\mathrm{lb} / \mathrm{ft}^{3}\right]$ :

$\rho, \quad$ density, two-phase mixture $(\rho=$ $\left.\rho_{g}+\rho_{l}\right)\left[\mathrm{lb} / \mathrm{ft}^{3}\right]$ :

$\rho c_{v}, \quad \rho c_{v} \equiv \rho_{g} c_{v_{g}}+\rho_{l} c_{l}$, equation (20):

$\rho_{g}$, density, vapor phase in mixture $\left(\rho_{g}=\alpha \bar{\rho}_{g}\right)\left[\mathrm{lb} / \mathrm{ft}^{3}\right]$ :

$\rho_{l}$, density, liquid phase in mixture $\left(\rho_{l}=(1-\alpha) \bar{\rho}_{l}\right)\left[\mathrm{lb} / \mathrm{ft}^{3}\right]$ :

$\bar{\rho}_{g}, \quad$ density, pure vapor phase $\left[\mathrm{lb} / \mathrm{ft}^{3}\right]$ :

$\bar{\rho}_{l}, \quad$ density, pure liquid phase $\left[\mathrm{lb} / \mathrm{ft}^{3}\right]$ :

$\sigma_{c}, \quad$ condensation coefficient [dimensionless] :

$\sigma_{e}$, vaporization coefficient [dimensionless] :

$\sigma, \quad$ mass transfer coefficient [dimensionless]: $\begin{array}{ll}\omega, & \text { frequency }[1 / \mathrm{s}] ; \\ \omega_{0}, & \text { resonant frequency for a bubble } \\ & {[1 / \mathrm{s}] .}\end{array}$

\section{INTRODUCTION}

THE PROPER treatment of several reactor safety problems requires a knowledge of how pressure waves propagate in a single-component, twophase mixture. For example, in a sodium cooled fast reactor, either a power excursion or a loss of coolant flow may initiate boiling in the sodium. A proper analysis of the sodium flow under these conditions requires a knowledge of the sound speed in the two-phase mixture [1].

A problem of interest in the safety studics of high pressure water cooled reactors is that of a reactor blowdown. It is well known that, in single-phase media, such as perfect gases, the critical flow rate is related to the sonic velocity [2]. It has been suggested that critical flow in a two-phase medium corresponds to a stationary pressure pulse [3]. Models developed for singlecomponent, two-phase media have generally included experimentally based correlations, particularly to describe non-equilibrium momentum transfer $[4,5]$. Most of these models require prior knowledge of the slip between the phases [3]. A model predicting the propagation of acoustic waves in two-phase mixtures should be of use in determining the proper manner to include nonequilibrium interphase effects in a critical flow model.

Experimental investigations into the propagation of pressure waves in two-phase, singlecomponent media have been limited to the study of large amplitude compression and rarefaction waves. The two-component studies of acoustic wave propagation as performed by Karplus [6] and by Mecredy et al. [7] have not been extended to single-component media.

Karplus [8] calculated the equilibrium sound speed in a steam-water mixture. He emphasized that an acoustic wave would travel at this speed only for sufficiently low frequencies. Based on the droplet or bubble size, Karplus evaluated a frequency limit below which the assumption of 
thermal equilibrium would be valid. Karplus also measured the speed of large amplitude waves and compared these data to a sound speed model which assumed equilibrium heat and momentum transfer between the phases, but no edge wave velocities were considerably larger than those predicted by the theory.

Other experimenters have also measured the two-phase, single-component media. An excellent summary of experimental results is given by Henry [9].

Predictions of the propagation speed of waves have generally been limited to models predicting the leading edge velocity of large amplitude waves. Fauske [10] developed a model using fitting parameters to analyse bubbly steam-water data. Henry [9] calculated the leading edge velocity as a function of void fraction using a correlation to account for the change in bubble shape with void fraction.

None of the models mentioned investigate the variation of either sound speed or attenuation with frequency in two-phase media. Marble has developed a model which predicts the attenuation of acoustic waves as a function of frequency in a condensing vapor fog $[11,12]$. The model predicts the sound speed only in certain limiting cases and assumes that the volume occupied by the liquid is negligible. mass transfer. In general, the measured leadingspeeds of large amplitude pressure waves in

In an earlier paper by the authors [13], the speed and attenuation of acoustic waves in bubbly two-component (e.g. air-water) media were determined, where the only nonequilibrium effect accounted for was interphase heat transfer. The model has been extended to include nonequilibrium heat, mass and momentum transfer between the two phases. The sound speed and attenuation are determined for two limiting flow patterns: vapor bubbles in continuous liquid and liquid droplets in continuous vapor.

\section{CONSERVATION EQUATIONS}

The most critical part of the analysis is the casting of the conservation equations in a form which properly describes the important nonequilibrium effects. The theoretical analysis of gas-solid systems by Soo [14] proved very helpful, especially with respect to the proper form of the nonequilibrium heat and momentum transfer terms. The conservation equations are:

\section{Conservation of mass}

$$
\begin{aligned}
& \frac{\partial \rho_{g}}{\partial t}+\frac{\partial}{\partial z}\left(\rho_{g} v_{g}\right)=\Gamma_{e}-\Gamma_{c} \\
& \frac{\partial \rho_{l}}{\partial t}+\frac{\partial}{\partial z}\left(\rho_{l} v_{l}\right)=\Gamma_{c}-\Gamma_{e} .
\end{aligned}
$$

Conservation of momentum

(a) Bubbles in continuous liquid

$$
\begin{aligned}
& \frac{\partial}{\partial t}\left(\rho_{g} v_{g}\right)+\frac{\partial}{\partial z}\left(\rho_{g} v_{g}^{2}\right)=-\alpha \frac{\partial p}{\partial z}+\rho_{g} F^{g l}\left(v_{l}-v_{g}\right)+\Gamma_{e} v_{l}-\Gamma_{c} v_{g}+\frac{1}{2} \bar{\rho}_{l} \alpha\left(\frac{1+2 \alpha}{1-\alpha}\right) \frac{\mathrm{D}}{\mathrm{D} t^{g}}\left(v_{l}-v_{g}\right) \\
& \frac{\partial}{\partial t}\left(\rho_{l} v_{l}\right)+\frac{\partial}{\partial z}\left(\rho_{l} v_{l}^{2}\right)=-(1-\alpha) \frac{\partial p}{\partial z}+\rho_{l} F^{l g}\left(v_{g}-v_{l}\right)+\Gamma_{c} v_{g}-\Gamma_{e} v_{l}-\frac{1}{2} \bar{\rho}_{l} \alpha\left(\frac{1+2 \alpha}{1-\alpha}\right) \frac{\mathrm{D}}{\mathrm{D} t^{g}} \\
& \quad \text { (b) Droplets in continuous vapor }
\end{aligned}
$$

$$
\begin{aligned}
\frac{\partial}{\partial t}\left(\rho_{g} v_{g}\right)+\frac{\partial}{\partial z}\left(\rho_{g} v_{g}^{2}\right)=-\alpha \frac{\partial p}{\partial z}+\rho_{g} F^{g}\left(v_{l}-v_{g}\right) & +\Gamma_{\mathrm{e}} v_{l} \\
& -\Gamma_{c} v_{g}+\frac{1}{2} \bar{\rho}_{g}(1-\alpha)\left(\frac{3-2 \alpha}{\alpha}\right) \frac{\mathrm{D}}{\mathrm{D} t}\left(v_{i}-v_{g}\right)
\end{aligned}
$$




$$
\begin{aligned}
\frac{\partial}{\partial t}\left(\rho_{l} v_{l}\right)+\frac{\partial}{\partial z}\left(\rho_{l} v_{l}^{2}\right)=-(1-\alpha) \frac{\partial p}{\partial z}+\rho_{l} F^{l g}\left(v_{g}-v_{l}\right) & +\Gamma_{c} v_{g} \\
& -\Gamma_{e} v_{l}-\frac{1}{2} \bar{\rho}_{g}(1-\alpha)\left(\frac{3-2 \alpha}{\alpha}\right) \frac{\mathrm{D}}{\mathrm{D} t^{l}}\left(v_{l}-v_{g}\right) .
\end{aligned}
$$

Conservation of energy

$$
\begin{array}{r}
\frac{\partial}{\partial t}\left[\rho_{g} U_{g}\left(T_{g}\right)+\frac{1}{2} \rho_{g} v_{g}^{2}\right]+\frac{\partial}{\partial z}\left[\rho_{g} U_{g}\left(T_{g}\right) v_{g}+\frac{1}{2} \rho_{g} v_{g}^{3}\right]=\rho_{g} c_{p g} G^{g l}\left(T_{l}-T_{g}\right)+\frac{\partial}{\partial z}\left[\alpha p v_{g}+(1-\alpha) p v_{l}\right] \\
+\Gamma_{e}\left[U_{g}\left(T_{l}\right)+p v_{f g}\left(T_{l}\right)\right]-\Gamma_{c}\left[U_{g}\left(T_{g}\right)+p v_{f g}\left(T_{g}\right)\right]-\frac{\rho_{g} c_{p g}}{\rho_{l} c_{l}+\rho_{g} c_{p g}}\left[\Gamma_{e} h_{g}\left(T_{l}\right)-\Gamma_{c} h_{f g}\left(T_{g}\right)\right] \\
\frac{\partial}{\partial t}\left[\rho_{l} U_{l}\left(T_{l}\right)+\frac{1}{2} \rho_{l} v_{l}^{2}\right]+\frac{\partial}{\partial z} \rho_{l} U_{l}\left(T_{l}\right) v_{l}+\frac{1}{2} \rho_{l} v_{l}^{3}=\rho_{l} c_{l} G^{l g}\left(T_{g}-T_{l}\right)+\Gamma_{c} U_{l}\left(T_{g}\right) \\
-\Gamma_{e} U_{l}\left(T_{l}\right)-\frac{\rho_{l} c_{l}}{\rho_{l} c_{l}+\rho_{g} c_{p_{g}}}\left[\Gamma_{e} h_{f g}\left(T_{l}\right)-\Gamma_{c} h_{f g}\left(T_{g}\right)\right] .
\end{array}
$$

The assumption has been made that the vapor is ideal, $\rho_{g}=\bar{\rho}_{g} R T_{g}$, and the liquid incompressible, $\bar{\rho}_{l}=$ constant. The equation of state is, therefore :

$$
\begin{aligned}
\rho_{l} & =\bar{\rho}_{l}(1-\alpha) \\
& =\bar{\rho}_{l}\left(1-\rho_{g} / \bar{\rho}_{g}\right) \\
& =\bar{\rho}_{l}\left(1-\frac{\rho_{g} R T_{g}}{p}\right)
\end{aligned}
$$

In considering the conservation equations, one should note the following:

1. The two-phase mixture has been treated as a pseudo single-phase fluid. When considering acoustic wave propagation, this assumption is valid provided the wavelength of the wave is much larger than the characteristic dimensions of the two-phase medium (e.g. the bubble diameter or average distance between bubbles) and provided the frequency of the wave is much less than any resonant frequency associated with the two-phase medium (e.g. for a single bubble,

$$
\left.\omega_{0}=\left(3 \gamma p / \bar{\rho}_{a} a^{2}\right)[15]\right) \text {. }
$$

2. $F^{g l}$ and $F^{l g}$ are the inverse time constants for momentum transfer from the liquid to the vapor and from the vapor to the liquid, respectively. Since, on a unit volume basis, the momentum lost through interphase momentum transfer by one phase must be gained by the other, one finds that

$$
\rho_{g} F^{g l}=\rho_{l} F^{l g} .
$$

3. The final term in each momentum equation accounts for the apparent mass of the vapor phase when accelerated relative to the liquid. According to Zuber [16], the induced mass of a spherical bubble in a mixture of vapor bubbles and liquid is :

$$
\frac{1}{2} \bar{\rho}_{l}\left(\frac{4}{3} \pi a^{3}\right)\left(\frac{1+2 \alpha}{1-\alpha}\right)
$$

The final term in this expression accounts for the effect of the surrounding bubbles on a given bubble.

4. $G^{g l}$ and $G^{l g}$ are the inverse time constants for interphase heat transfer. Energy lost by one phase through interphase heat transfer must be gained by the other, resulting in the relationship

$$
\rho_{g} c_{p g} G^{g l}=\rho_{l} c_{l} G^{g g}
$$

5. Since the liquid is incompressible, all flow work performed on the mixture must be performed on the vapor phase. This includes work done when phase change takes place.

6. The heat of vaporization required for evaporation is drawn from each phase in proportion to its heat capacity per unit volume of 
mixture. At high frequencies, the heat drawn from each phase should be related to the thermal diffusivities of the two media, however, in either case, most of the heat required for vaporization comes from the liquid.

7. Viscous forces and heat conduction within each phase are assumed small. In studies with two-phase, two component media [13], it was found that ignoring these effects resulted in an error in sound speed or attenuation of less than 0.5 per cent. Viscous dissipation is neglected in the energy equations. These terms, if included, vanish when the conservation equations are linearized.

\section{EVALUATION OF NONEQUILIBRIUM TERMS}

The frequency dependence of both the sound speed and attenuation depends very strongly on the values of $\Gamma_{e}$ and $\Gamma_{c}$, the source and sink terms for mass transfer; $F^{g^{i}}$, the inverse time constant for interphase momentum transfer; and $G^{g l}$, the inverse time constant for interphase heat transfer.

\section{Mass transfer}

The evaporation and condensation processes must be considered separately because the liquid is evaporating at velocity $v_{t}$ and temperature $T_{l}$ while the condensation occurs at $v_{y}$ and $T_{g}$. This results in additional interphase heat and momentum transfer. From kinetic theory [17] one may write, for the mass transfer per unit volume:

(a) Bubbles in continuous liquid

$$
\Gamma_{e}=\frac{3 \sigma_{e}^{\alpha}}{a \sqrt{2 \pi R}} \frac{p^{*}}{\sqrt{ } T_{l}}
$$

and

$$
\Gamma_{c}=\frac{3 \sigma_{c} \alpha}{a \sqrt{ } 2 \pi R} \frac{p}{\sqrt{ } T_{g}} .
$$

(b) Droplets in continuous vapor

$$
\Gamma_{e}=\frac{3 \sigma_{e}(1-\alpha)}{a \sqrt{ } 2 \pi R} \frac{p^{*}}{\sqrt{ } T_{l}}
$$

and

$$
\Gamma_{\mathrm{c}}=\frac{3 \sigma_{c}(1-\alpha)}{a \sqrt{2 \pi R}} \frac{p}{\sqrt{T_{g}}} .
$$

In the analysis, the evaporation coefficient $\sigma_{e}$ and the condensation coefficient $\sigma_{c}$ are assumed equal,

$$
\sigma_{e}=\sigma_{c}=\sigma .
$$

The use of one of the more detailed mass transfer models, such as that proposed by Patton and Springer [18], is not warranted since there is no agreement in the literature as to the values to be used for $\sigma_{e}$ and $\sigma_{c}$. For example, there is some indication that the condensation coefficient decreases with increasing system pressure [19]. It is also felt that system contamination will reduce the mass transfer coefficients from the values obtained in a "clean" system [20].

\section{Momentum transfer}

The force exerted on a bubble by the surrounding liquid due to relative motion may be readily calculated for sufficiently small bubbles and for Stokes flow. For bubble radii less than about 03 inches, the bubbles may be treated as solid spheres in the calculation of $F^{g t}$ [21]. Under these assumptions, the force on the bubble caused by the surrounding liquid moving at velocity $U$ relative to the bubble is [22] :

$$
F_{B}=6 \mu \pi a U .
$$

In a mixture with void fraction $\alpha$, the force per unit volume of the mixture is found to be

$$
N \times F_{B}=\left(\frac{\alpha}{\frac{4}{3} \pi a^{3}}\right)(6 \mu \pi a U)=\frac{9}{2} \alpha \frac{\mu}{a^{2}} U
$$

where $N$ is the number of bubbles per unit volume. This force may also be expressed as

$$
\alpha \bar{\rho}_{g} \frac{\partial v_{g}}{\partial t}=\alpha \bar{\rho}_{g} F^{g l}\left(v_{l}-v_{g}\right)
$$

Comparing equations (15) and (16), one obtains an expression for $F^{g t}$

$$
F^{g l}=\frac{9}{2} \frac{\mu}{\bar{\rho}_{g} a^{2}} .
$$


For the case of droplets in continuous vapor, again with the assumption of Stokes flow, the expression for $F^{g l}$ is

$$
F^{g l}={ }_{2}^{9}\left(\begin{array}{c}
1-\alpha \\
\alpha
\end{array}\right) \begin{gathered}
\mu \\
\bar{\rho}_{g} a^{2}
\end{gathered} .
$$

\section{Heat transfer}

If, in the case of bubbly flow, it is assumed that the major resistance to interphase heat transfer is conduction within the vapor phase, the inverse time constant for heat transfer from the liquid to the vapor is found to be frequency dependent and is given by [13]

$$
G^{g l}(\omega)=G_{0}^{g l} R e\left\{\frac{\beta \operatorname{coth} \beta-1}{5-\frac{15}{\beta^{2}}(\beta \operatorname{coth} \beta-1)}\right\}
$$

where

$$
\begin{gathered}
\beta^{2}=i \omega a^{2} / K_{g} \\
G_{0}^{g l}=G^{g l}(0)=\frac{15 K_{g}}{a^{2}}
\end{gathered}
$$

$G^{g l}(\omega)$ is shown as a function of frequency in Fig. 1.

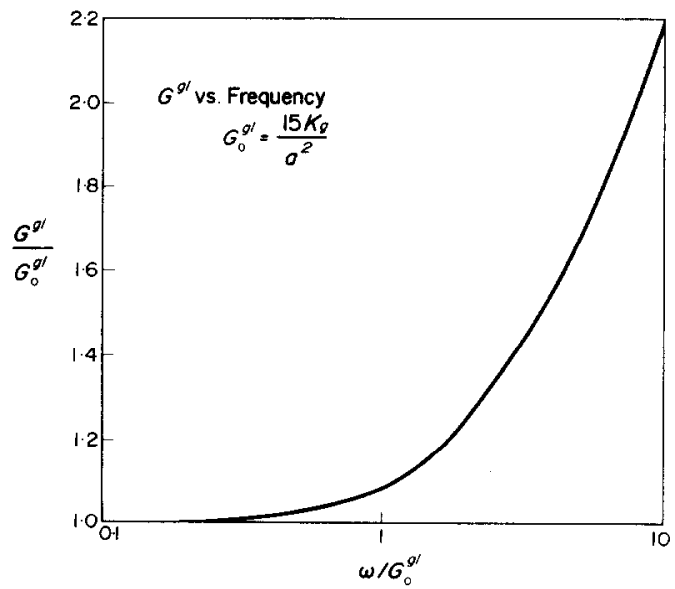

Fig. 1. Inverse time constant for heat transfer from the liquid phase to the vapor phase as a function of frequency in a bubbly steam-water mixture.

For the case of droplets in continuous vapor, the major resistance to interphase heat transfer occurs in the vapor boundary layer. In the limit of low Reynolds number, Soo [14] has shown that $G^{g l}$ is given by

$$
G^{g l}=\left(\begin{array}{c}
1-\alpha \\
\alpha
\end{array}\right) \begin{gathered}
3 K_{g} \\
a^{2}
\end{gathered}
$$

\section{DETERMINATION OF SOUND SPEED AND ATTENUATION}

The set of conservation and state equations [equations (1), (2), (3a), (4a), (5), (6) and (7) for bubbles in continuous liquid or equations (1), (2), (3b), (4b), (5), (6) and (7) for droplets in continuous vapor] are linearized to obtain the acoustic equations for the medium.

A Laplace transform in the time variable, with $s=i \omega$, and a Fourier transform in the space variable are then performed. This is equivalent to assuming that each dependent variable can be represented as $A \mathrm{e}^{i(k z+\omega t)}$, where $A$ is some complex constant and $k=(\omega / c)+i \eta$. A set of

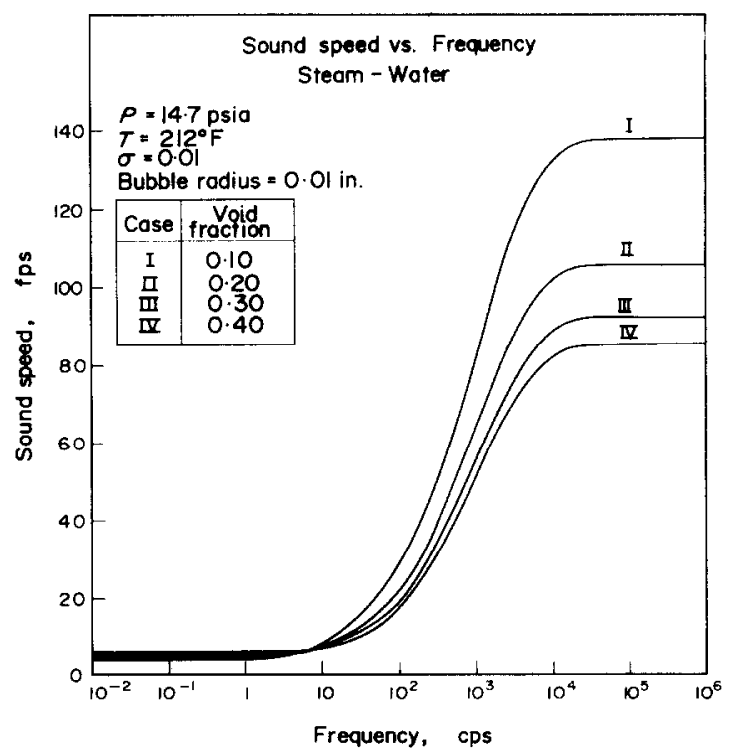

FIG. 2. Sound speed as a function of frequency in a bubbly steam-water mixture.

seven homogeneous equations in seven unknowns is obtained; for a unique solution to exist, the determinant of the coefficient matrix must vanish. For example, in the case of bubbles in continuous liquid, this implies that: 


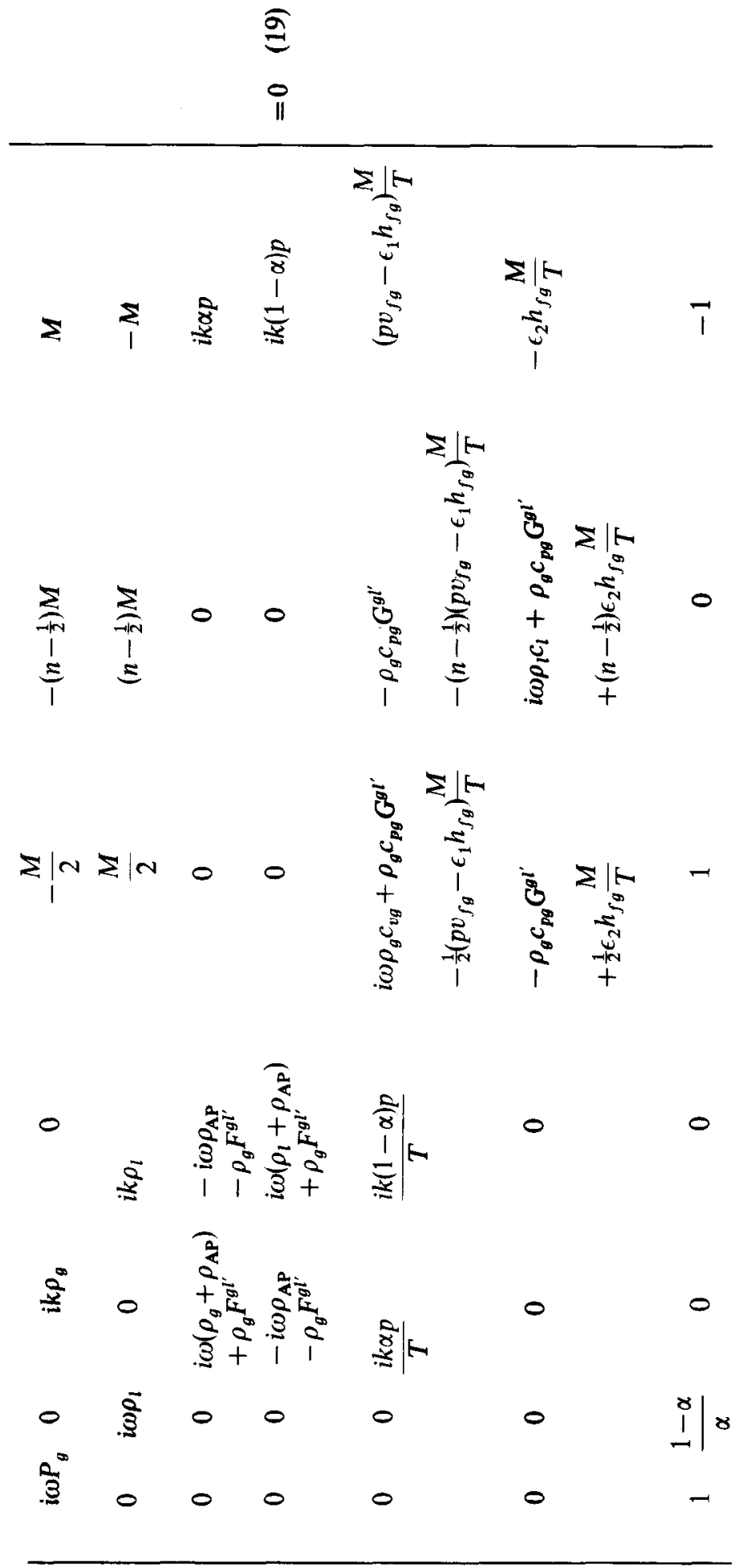


A similar determinant is obtained for the case of droplets in continuous vapor.

Expanding the determinant yields a quadratic equation in $k$ which is solved numerically by means of a Laguerre iteration technique. The two roots are equal but opposite in sign, one corresponding to a wave moving in a positive $z$ direction and the other in the negative direction. The real part of $k$ is then used to obtain the sound speed $[\operatorname{Re}(k)=\omega / c)]$, while the imaginary part of $k$ equals $\eta$, the attenuation coefficient.

\section{RESULTS AND DISCUSSION}

The sound speed is shown as a function of frequency for bubbles in continuous liquid in Figs. 2 and 3, and for droplets in continuous

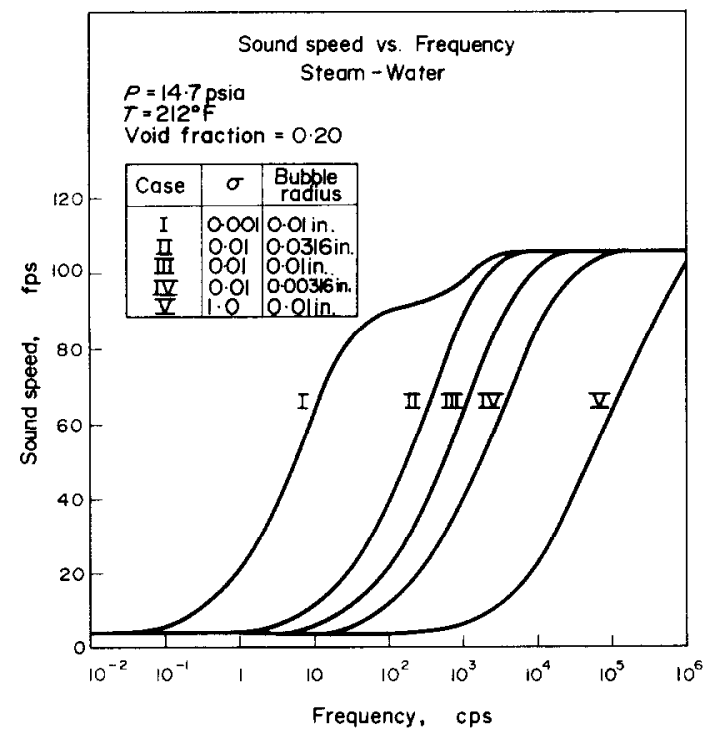

Fig. 3. Sound speed as a function of frequency in a bubbly steam-water mixture.

vapor in Figs. 4 and 5. There are several important factors to be noted :

1. In all cases, the low frequency limit of the sound speed is that calculated assuming mechanical and thermal equilibrium in the mixture;

$$
\begin{aligned}
\lim _{\infty \rightarrow 0} c^{2}=\left(\frac{\partial p}{\partial \rho}\right)_{s}= & \frac{1}{\alpha \rho}\left\{\left(\frac{U_{f g}}{h_{f g}}\right)^{2} \frac{1}{p}\right. \\
& \left.+\frac{\rho c_{v} T}{\alpha}\left(\frac{v_{f g}}{h_{f g}}\right)^{2}\right\}^{-1} .
\end{aligned}
$$

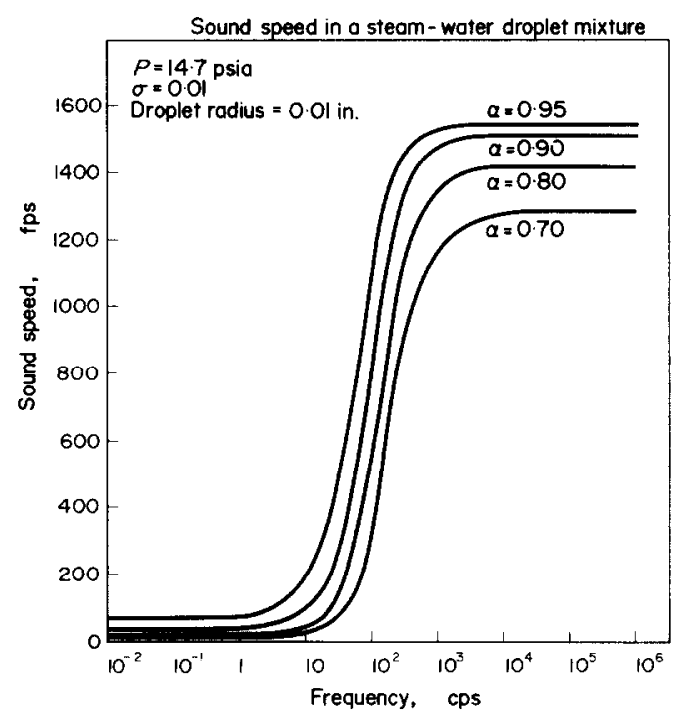

FIG. 4. Sound speed as a function of frequency in a steamwater droplet mixture.

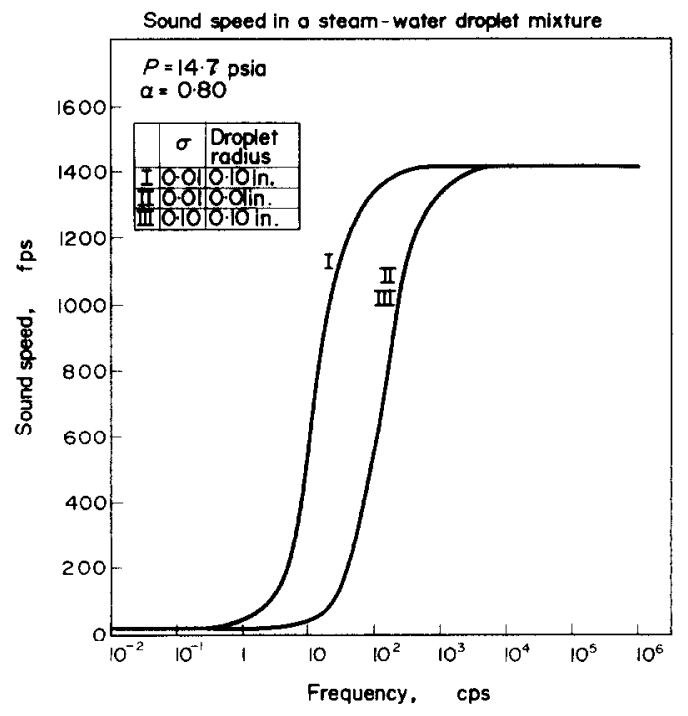

FIG. 5. Sound speed as a function of frequency in a steamwater droplet mixture. 
2. On the other hand, the high frequency limit of the sound speed is that obtained assuming no heat, mass, or momentum transfer between the phases;

(a) Bubbles in continuous liquid

$$
\lim _{\infty} c^{2}=\frac{\gamma p}{\alpha \rho}\left\{1+\frac{2 \alpha(1-\alpha)^{2}}{1+2 \alpha}\right\}
$$

(b) Droplets in continuous vapor

$$
\lim _{i \rightarrow \infty} c^{2} \cong \frac{\gamma p}{\bar{\rho}_{g}}\left\{\frac{\alpha}{\alpha+\frac{(3-2 \alpha)(1-\alpha)}{2 \alpha}}\right\}
$$

3. As seen in Figs. 3 and 5 , the frequency at which non-equilibrium effects become important is a strong function of the bubble or droplet radius and the mass transfer coefficient. Since the evaporation and condensation rates are proportional to $\sigma / a$ [see equations (11) and (12)], while the inverse time constants for interphase heat and momentum transfer are proportional to $1 / a^{2}$ [see equations (17) and (18)], equilibrium

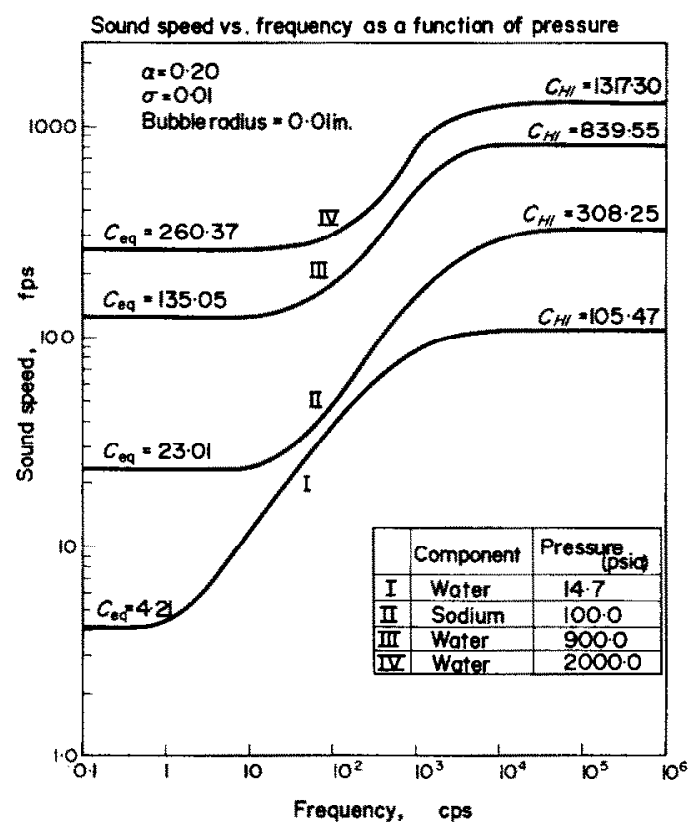

Fig. 6. Sound speed as a function of frequency in bubbly two-phase, single-component media. can be maintained in the mixture at higher frequencies as either $\sigma$ increases or $a$ decreases.

The sound speed has been plotted as a function of frequency in Fig. 6 for conditions of interest for nuclear reactor applications. Calculations have been performed at a void fraction of 20 per cent for steam-water mixtures at pressures of $14 \cdot 7,900$ and 2000 psia and for sodium-sodium vapor at 100 psia. As can be seen in Fig. 6, an increase in pressurc causes an incrcase in sound speed with the high frequency limit behaving as $p^{\frac{1}{2}}$ [see equation (21a)]. The speed of an acoustic wave in a sodium-sodium vapor mixture at 100 psia and 20 per cent void fraction is seen to increase by a factor of ten from the low to the high frequency limits. Thus, in sodium systems, as well as water systems, frequency dependence must be included when considering wave propagation.

Wave attenuation as a function of frequency is shown for bubbly steam-water mixtures in Fig. 7 and for water droplets in continuous

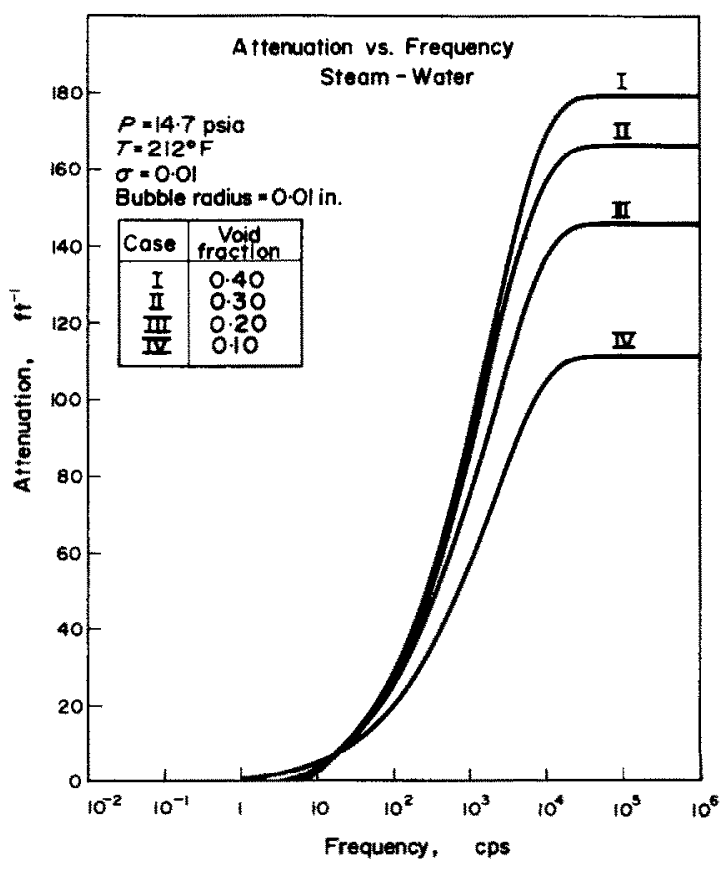

FiG. 7 Attenuation coefficient as a function of frequency in a bubbly steam-water mixture. 
vapor in Fig. 8. In all cases the attenuation coefficient goes to zero in the low frequency limit where equilibrium is maintained in the two-phase mixture. In the high frequency limit, the attenuation coefficient approaches a constant

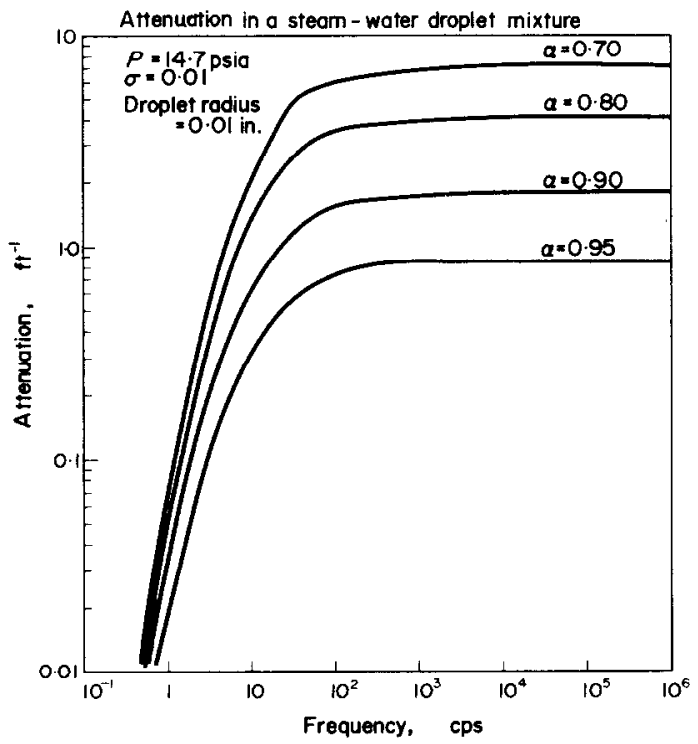

FIG. 8. Attenuation coefficient as a function of frequency in a steam-water droplet mixture.

value. This limiting value tends to increase with increasing void fraction. As seen in Fig. 7, at moderate void fractions and high frequencies the attenuation can be very strong. For example, at a frequency of $10 \mathrm{kHz}$ and void fraction of 40 per cent, the attenuation coefficient equals $170 \mathrm{ft}^{-1}$. This means that the wave will attenuate by a factor of " $e$ " in (1/170) $\mathrm{ft}$ which is less than one tenth of an inch.

As was mentioned in the introduction, measurements of either sound speed or attenuation in single-component mixtures have not been performed. The very large wave attenuations expected certainly make the design of such an experiment more difficult. A comparison of the theory, at least in part, can be made to the experimentally measured wave velocities of large amplitude pressure waves (for a summary of these experimental results, see [9]). In all

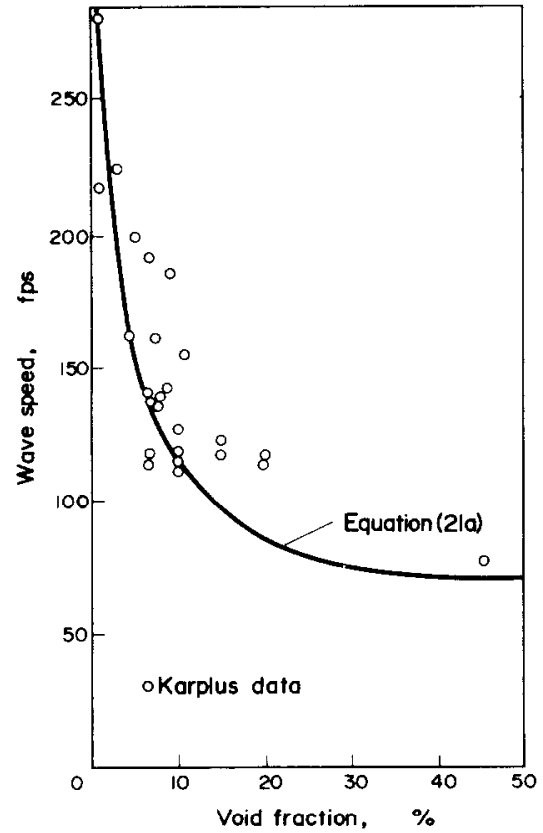

FIG. 9. Comparison of Karplus' leading edge data [8] with theory.

cases the large amplitude waves were found to broaden as they propagated; this means that each point on the wave travelled with respect to the fluid at the local sound speed. In addition, since sound speed increases with frequency, it is expected that the leading edge of the wave travelled at the high frequency limit of the sound speed. This conclusion is reached since a finite amplitude wave can be thought of as the superposition of a large number of waves of various frequencies. The high frequency components travel with the highest speeds (see Figs. 2-6) and thus form the leading edge of the wave. In Fig. 9, the theoretical expression for the high frequency limit of the sound speed [equation (21a)] is compared to the bubbly steam-water data of Karplus [8], while in Fig. 10 the high void fraction steam-water data of Collingham and Trapp [23] and England et al. [24] are compared to equation (21b). In order to write equation (21b) in terms of quality rather than void fraction, the slip ratio in the unperturbed 


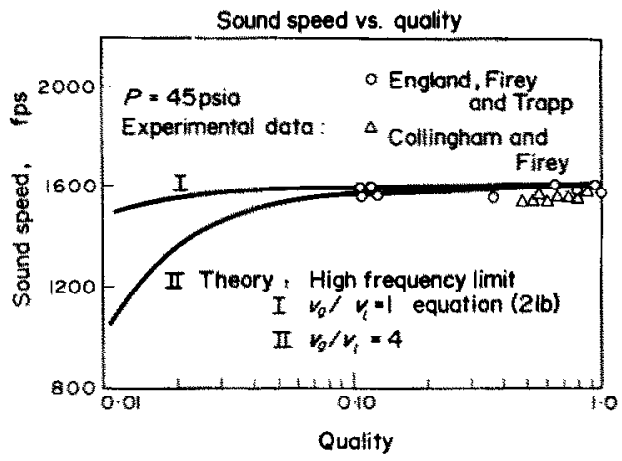

Fia. 10. Comparison of data from vapor-continuous twophase media $[23,24]$ with theory.

mixture must be known. Theoretical curves of sound speed vs. quality are shown in Fig. 10 for slip ratios of 10 and 40 . In both Fig. 9 and Fig. 10 the agreement between theory and experiment is good.

\section{SUMMARY}

In summary, calculations have shown that both the sound speed and the sound attenuation are strong functions of frequency in two-phase, single-component (liquid-vapor) mixtures. At high frequencies and low void fractions the attenuation can be extremely large. A comparison with experimental data shows that the leading edges of large amplitude pressure waves travel at the high frequency limit of the sound speed.

\section{ACKNOWLEDGEMENTS}

One of the authors (RCM) is grateful for the financial assistance received from a USAEC fellowship in Nuclear Science and Engineering and from a Michigan Memorial Pheonix Project fellowship. The second athor (LJH) would like to acknowledge support received from a National Science Foundation grant (GK-4989). The authors appreciate the support for computing provided by the University of Michigan Computing Center.

\section{REFERENCES}

1. E. R. SiEgMaNN, A compressible model for transient sodium boiling, Trans. Am. Nucl. Soc. 12, 904 (1969).

2. A. H. ShapIRo, The Dynamics and Thermodynamics of Compressible Fluid Flow, Vol. 1. Ronald Press, New York (1953).

3. F. J. Moody, A pressure pulse model for two-phase critical fow and sonic velocity, $J$. Heat Transfer 91 , $371-384(1969)$.

4. K. FAUSKe, Contribution to the theory of two-phase one-component critical flow, ANL-6633 (1962).
5. R. E. HenRy, H. K. Fauske and S. T, McComas, Twophase critical flow at low qualities. Part II: Analysis, Nucl. Sci. Engng 41, 92-98 (1970).

6. H. B. Karplus, The velocity of sound in a liquid containing gas bubbles, COO-248, Armour Research Foundation (1958).

7. R. C. Mecredx, J, M. Wigdortz and L. J. Hamilton, Prediction and measurement of acoustic wave propaga tion in two-phase media, Trans. Am. Nucl, Soc. 13, $672(1970)$.

8. H. B. Karplus, Propagation of pressure waves in a mixture of water and steam, ARF4432, Armour Research Foundation (1961).

9. R. E. Henry, Pressure wave propagation in two-phase mixtures, Proc. Eleventh Nat'l. Heat Transfer Conf. (1969).

10. H. K. Fauske, Propagation of pressure disturbances in two-phase flow, Proc. Symp. on the Dynamics of Two Phase Flow at the University of Eindhoven, Nether lands (1967).

11. F. E. MARBLE, Some gas dynamic problems in the flow of condensing vapors, Astronautica Acta 14, 585-614 (1969).

12. F. E. MARBLE and D. C. Wooten, Sound attenuation in a condensing vapor, Physics Fluids 13, 2657-2664 (1970).

13. R. C. MECREDY and L. J. Hamilton, Speed and attenuation of acoustic waves in two-phase two-component media, Proc. Sixth Southeastern Seminar Thermal Sciences (April 1970).

14. S. L. Soo, Fluid Dynamics of Multiphase Systems, Ch. 6. Blaisdel, Waltham, Mass. (1967).

15. M. MinnaekT, On musical air bubbles and the sounds of running water, Phil. Mag. 16, 235 (1933).

16. $N_{x}$ ZUBER, On the dispersed two phase flow in the laminar flow regime, Chem. Engng Sci. 19, 897 (1964).

17. E. H. KenNaRd, Kinetic Theory of Gases. McGraw-Hill (1938).

18. A. J. Patton and G. S. Springer, A kinetic theory description of liquid vapor phase change, Proc. Sixth Int. Symp. on Rarified Gas Dynamics, 2, 1497-1501 (1969).

19. I. T. AladyeV, N. S. Kondratyev, V. A. Mukhn, M. E. Kipshidze, I. Parfentyeva and V. V. Kisselev, Thermal resistance of phase transition with condensation of potassium vapor, Proc. Third Int'l. Heat Transfer Conf., 313-317 (1966).

20. K. F. WYLE and C. E. DRYDEN, Transport phenomena at liquid-metal-vapor interfaces using radioactive tracers, Nucl. Appl. 5, 263-268 (1968).

21. G. B. Wallis, One-Dimensional Two-Phase Flow. McGraw-Hill, New York (1969).

22. SiR Horace LamB, Hydrodynamics, Sixth Ed., p. 598. Dover, New York (1945).

23. R. E. Collingham and J. C. Firey, Velocity of sound measurements in wet steam, I/EC Proc. Des. Dev. 2(3), 197-202(1963).

24. W. G. ENGland, J. C. Firey and O. E Trapp, Additional velocity of sound measurements in wet steam, I/EC Proc. Des. Dev. 5 (2), 198-202 (1966). 


\section{LES EFFETS D'UN TRANSFERT HORS D'ÉQUILIBRE DE MASSE, DE CHALEUR FT DF QUANTITE DE MOUVEMENT SUR LA CÉLERITE DU SON DANS UN MILIEU BIPHASIQUF.}

Résumé-Il a été développé un modèle qui donne la célérité et l'atténuation des ondes acoustiques comme étant fonction de la fréquence dans un milieu biphasique à un seul composant. L'absence d'équilibre entre phase pour le transfert de masse, de chaleur et de quantité de mouvement a été pris en compte et a été trouvé important. Aux basses fréquences la vitesse de l'onde approche la valeur d'équilibre calculée par $\sqrt{ }(\partial p / \partial \rho)_{s}$. A la limite, dans les hautes fréquences la célérité de l'onde est caractérisée par l'absence de transfert de masse, de chaleur et de quantité de mouvement entre les phases liquide el vapeur. L'alténuation est grande aux hautes fréquences. Une comparaison entre théorie et expérience montre que les frontières antérieures des ondes de pression de grande amplitude se déplacent à la célérité du son correspondant à la limite des hautes fréquences.

\section{DER EINFLUSS VON WÄRME-, STOFF- UND IMPULSÜBERTRAGUNG IM NICHT-GLEICHGEWICHT AUF DIE ZWEI-PHASEN-SCHALLGESCHWINDIGKEIT}

Zusammenfassung-Ein Modell wurde entwickelt, das Geschwindigkeit und Dämpfung akustischer Wellen liefert als Funktion der Frequenz in zweiphasigen Ein-Komponenten-Medien. Wärme-, Stoffund Impulsübergang an der Nicht-Gleichgewichtsphasengrenze wurde berücksichtigt und als wichtig befunden. Bei niedrigen Frequenzen erreicht die Wellengeschwindigkeit den Gleichgewichtswert $\sqrt{ }\left[(\partial P / \partial \rho)_{s}\right]$. Die Schallgeschwindigkeit bei hohen Frequenzen ist durch das Fehlen von Wärme-, Stoffoder Impulsübergang zwischen der flüssigen und dampförrmigen Phase gekennzeichnet. Die Dämpfung ist bei hohen Frequenzen gross. Ein Vergleich der Theorie mit dem Experiment zeigt. dass sich die Vorderfront von Druckwellen grosser Amplituden mit dem Hochfrequenzspitzenwert der Schallgeschwindigkeit fortgewegt.

\section{ВЛИЯНИЕ НЕРАВНОВЕСНОГО ПЕРЕНОСА ТЕПЛА, М АССЫ И КОЛИЧЕСТВА ДВИЖЕНИЯ НА ДВУХФАЗНУЮ СКОРОСТБ ЗВУКА}

\footnotetext{
Аннотадня-Равработана модель, позволяющая получить скорость и затухание акустических волн как функцию частоты в двухфазных однокомпонентных средах. Показано, что неравновесный межфавовый перенос тепла, массы и количества движения вначительно влияет на скорость акустических волн. При низких частотах скорость волны достигает равновесного значения, рассчитанного по $\sqrt{ }\left[(\partial P / \partial \rho)_{s}\right]$. В предельном случае высокой частоты скорость волны характеризуется отсутствием переноса тепла, массы или количества движения между жидкой и паровой фазами. Затухание большое при высоких частотах. Сравнение теории с акспериментом показывает, что передний фронт волн с большой амплитудой давления проходит при высоком пределе частоты скорости звука.
} 